\title{
Complex diffractometrical investigation of structural and compositional irregularities in GaAs:Si/GaAs films heavily doped with silicon
}

\author{
L.I. Datsenkoa)，V.P. Klad'koa)，P.M. Lytvyn ${ }^{\text {a) }}$ ，J. Domagalab)， V.F. Machulin ${ }^{\text {a) }}$, \\ I.V. Prokopenko a), V.B. Molodkin' ${ }^{\mathrm{c}}$, Z.V. Maksimenko ${ }^{\text {a) }}$ \\ a) - Institute of the Semiconductors Physics, National Academy of Sciences of Ukraine, Prospekt Nauki 45, 03028 Kyiv, Ukraine \\ b) - Institute of Physics, Polish Academy of Sciences, Al.Lotnikow 32/46, 02-668 Warsaw, Poland. \\ c) - G.V. Kurdyumov Institute for Metal Physics, National Academy of Sciences of Ukraine, Vernadskogo blvd. 36, 03680, \\ Kyiv142, Ukraine
}

\begin{abstract}
Complex of X-ray diffractometrical investigations, both angular and spectral dependences of a reflectivity for quasi-forbidden reflections, enable not only to discover structural microdefects and to measure their radii $r$ as well as concentration $n$, but also to determine the level of nonstoichiometry, $\Delta=C_{A}-C_{B}$, where $C_{A}, C_{B}$ are concentrations of lattice components $A$ and $B$, respectively. In the case of angular dependencies, the twodimensional maps of diffuse scattering in a reciprocal space for a characteristic radiation were plotted for GaAs:Si/GaAs films heavily doped by Si (up to $10^{20} \mathrm{~cm}^{-3}$ ) using a three-crystal spectrometer (TCS). In the case of spectral (energy) dependencies, reflectivity were measured using a single crystal spectrometer (SCS) and white beam radiation. In both cases the formulae of the Molodkin dynamical scattering theory developed for real crystals with homogeneously distributed microdefects were used by the fitting procedure of the calculated intensities to those measured using TCS or SCS for (200) and (400) reflections of X-rays. The TCS maps were registred for $\mathrm{Cu} K_{\alpha}$ - radiation by the Phillips three-crystal diffractometer.

Good agreement between the two groups of the $\bar{r}$ and $\bar{n}$ parameters of microdefects (precipitates) was shown for some GaAs film $\left(r_{1}=3.5 \mu \mathrm{m}, n_{1}=4.3 \cdot 10^{6} \mathrm{~cm}^{-3} ; r_{2}=4.8 \mu \mathrm{m}, n_{2}=9.4 \cdot 10^{6} \mathrm{~cm}^{-3}\right)$. Parameter $\Delta=0.009$ (Ga excess) was determined too.
\end{abstract}

Keywords: X-ray diffraction, point defects, triple crystal diffractometer, GaAs.

Paper received 18.01.01; revised manuscript received 25.01.01; accepted for publication 13.07.01.

\section{Introduction}

Diffuse scattering (DS) of shortwave radiation is widely used nowadays for investigation of structure defects in crystals [1-13]. The theories of DS have been developed and generalized by many authors [1,5-7]. The double (DCS) as well as three crystal (TCS) spectrometers are applied for experimental investigations of the DS in such objects. The last one is used in the so-called diffractometry of high resolution.

A TCS permits to study the distribution of intensity in the reciprocal space near the reciprocal lattice points (RELP). New informative and sensitive approach in the three-crystal diffraction theory was recently developed [14]. In this work the important relations between integral and differential intensities of the DS observed usually in the profiles of intensity were proposed.
GaAs crystals heavily doped with Te, Se, Si and other impurities have no or little dislocation [15]. The behavior of point-like defects in such crystals, i.e. the so-called Coulomb microdefects (precipitates or dislocation loops), with deformation fields quadratically depending on the distance from a center is of interest for investigation by analysis of the DS peculiarities. So the aim of this paper was investigation of the real structure in the GaAs films heavily doped with Si by means of analysis of the DS pictures registered by high resolution diffractometry for usual and quasi-forbidden reflections (QFRs). To obtain the independent values of the above-mentioned parameters of a defect structure as well as the level of nonstoichiometry, $\Delta$, the analysis of the spectral dependence of total integral reflectivity (IR), $R_{i}$, of the (200) QFR for the wavelengths situated between the Ga and As absorption $K$-edges [16] was also planned to carry out. 


\section{L.I. Datsenko et al.: Complex diffractometrical investigation of structural and ...}

\section{Theoretical basis}

Reflectivity of a real crystal is known to depend on the diffraction characteristics which are the Debye-Waller static factor $E=\exp \left(-L_{H}\right)$ and the extinction coefficient $\mu_{d}$ due to diffuse scattering on defects. The last parameter was also introduced to take into account the dynamical effects in a diffuse scattering $\mu_{d} *$ [14]. It is important to stress here that all of these parameters are integral ones and depend on the concentation $c$ of all defects $N$ in a crystal as well as on a displace field $u_{t s}$ created in each lattice node $s$ by a defect in position $t[1,14,18]$ :

$$
\begin{aligned}
& L_{H}=1 / 6 \mathrm{c} \cdot \sum_{i=1}^{N}\left\langle u_{t s}^{2}\right\rangle_{i}, \\
& \mu_{d}=c^{2} v / 4 \lambda^{2} \cdot \int_{E . S} d \sigma(q) / d \Omega \cdot d S(q) .
\end{aligned}
$$

Here $v, \lambda, q$ respectively stand for a volume of an elementary cell, wavelength of X-rays, distance from the nearest reciprocal-lattice point $H$ in reciprocal space where the diffuse scattering is measured. The integral in (2) is taken over the Ewald sphere (ES) and $d \sigma(q) / d q$ is the differential cross section for diffuse scattering function depending on the Fourier coefficient of susceptibility $\chi$ of a crystal, the Debye-Waller factor $E=\exp \left(-L_{H}\right)$ and on the Fourier component of the distortion field of an individual defect.

The expression for the coherent component of the reflection coefficient $R_{c o h}(\Delta \vartheta)$ of a sample with homogeneous distribution of defects [14], used in the spectral dependence of $R_{i}$ method [16], has the following form:

$$
R_{c o h}(\Delta \vartheta)=\zeta \cdot\left(L-\sqrt{L^{2}-1}\right)
$$

Here $L$ is a function of different scattering parameters which describe the absorption of coherent waves due to inelastic scattering processes (photoelectric absorption, Compton effect, thermal diffuse scattering). The parameters $h, d$ and $a$ take into account the additional absorption of the coherent waves due to the diffuse scattering on defects, characterized by $\mu_{d s}$ and $\mu_{d s}{ }^{*}$. If the dispersion corrections for the Fourier coefficients of a susceptibility $c$ remained in the approximation of semiinfinite crystal $\Delta \chi_{G G^{\prime}}^{\delta}=P_{G G^{\prime}}^{\delta}-i \mu_{G G^{\prime}}^{\delta} / K$ are denoted as $p_{G G^{\prime}}=$ $=\operatorname{Re} P_{G G^{\prime}}^{\delta} /(C E)$ and $m_{G G^{\prime}}=-\operatorname{Re} \mu_{G G^{\prime}}^{\delta} /(K C E)$ and the approximate equalities $p_{o h} \approx p_{h 0}$ and $m_{0 h} \approx m_{h 0}$ hold, then these parameters can be represented as

$$
\begin{aligned}
& h=\left(m_{h h}+m_{00} / b_{S}\right) \sqrt{b_{S}} /\left(2 C\left|\chi_{r h}\right|\right), \quad d=m_{h 0} \chi_{r h}^{\prime} /\left|\chi_{r h}\right|^{2}, \\
& a^{2}=\left(m_{h h}^{2}-p_{h 0}^{2}-\chi_{r h}^{\prime} p_{h 0}\right) /\left|\chi_{r h}\right|^{2}, \quad \chi_{r h}^{\prime}=\operatorname{Re} \chi_{r h} .
\end{aligned}
$$

The diffuse component of the reflection coefficient $R_{D S}(\Delta \vartheta)$ of the crystal with homogeneously distributed defects after integration it over the output angles can be represented [14] as

$$
R_{D}(\Delta \vartheta)=F_{d y n}(\Delta \vartheta) \cdot \frac{\mu_{d s}\left(k_{0}\right) \cdot \gamma_{0}}{2 \cdot \mu_{i}(\Delta \vartheta)}
$$

$F_{d y n}(\Delta \vartheta)$ is an extinction factor describing the modulation of diffuse scattering intensity due to extinction of the strong Bragg waves.

$$
\begin{aligned}
& \mu_{d s}\left(k_{0}\right)=n \cdot C^{2} \cdot E^{2} \cdot m_{0} \cdot J_{0}\left(k_{0}\right), \\
& m_{0}=\pi \cdot a^{3} \cdot\left(H \cdot\left|\Psi_{h}\right| / \lambda\right)^{2} / 4, \\
& J_{0}\left(k_{0}\right)=b_{2} \cdot \ln \frac{e \cdot\left(k_{m}^{2}+\mu_{i}^{2}\right)}{k_{o}^{2}+\mu_{i}^{2}}+b_{3} \cdot\left(\frac{k_{0}^{2}}{2 \cdot k_{m}^{2}}-1\right), \\
& \text { for }\left|k_{0}\right| \leq k_{m} \\
& J_{0}\left(k_{0}\right)=\left(b_{2}-\frac{1}{2} \cdot b_{3}\right) \cdot \frac{k_{m}^{2}}{k_{0}^{2}}, \text { for }\left|k_{0}\right| \geq k_{m} .
\end{aligned}
$$

$H, r, n$ stand for the reciprocal lattice vector, $n$ concentracion of defects, and the defect radius respectively, $e$ here is the base of natural logarithm, $k_{0}=$ $=K \cdot \Delta \vartheta \cdot \sin (2 \vartheta), k_{m}=2 \cdot \pi / r_{0}, b_{2}=B_{1}+B_{2} \cdot \cos ^{2}(\vartheta / 2)$, $b_{3}=B_{2} \cdot \cos ^{2} \vartheta \cdot\left(1-2 \cdot \operatorname{tg}^{2} \vartheta\right) / 2$. For the spherical cluster model $[17,18]$, the following expressions hold: $B_{1}=0$, $B_{2}=\left(4 \cdot \pi \cdot A_{c l} / a^{3}\right)^{2}, A_{c l}=\Gamma \cdot \varepsilon \cdot r_{0}^{3}, \Gamma=(1+v) /[3 \cdot(1-$ $-v)], \varepsilon$ is the deformation parameter and $v$ denotes the Poisson ratio.

\section{Experimental}

The two-dimensional maps of the intensity distribution for $\mathrm{GaAs}$ films heavily doped with $\mathrm{Si}$ (with concentration up to $10^{20} \mathrm{~cm}^{-3}$ ) were measured using the three-crystal Philips diffractometer at the Institute of Physics PAS, Warsaw. The $\Delta \vartheta$ profiles, i.e. the curves of diffraction reflection (CDR) obtained without an analyser (the case of DCS) as well as $\vartheta-2 \vartheta$ profiles at fixed positions of a sample $\Delta \vartheta_{i}$, were taken for the (200) and (400) reflections. The contribution of diffuse scattering intensity $I_{D S}$ in every point of the CDR was determined by subtraction of the calculated value of intensity $I_{P}$ for a perfect crystal from the experimental values $I_{\text {exp }}$, i.e. $I_{D S}=I_{\text {exp }}-I_{P}$. The symmetrical part of the DS, $I_{S}=\left[I_{D S}\left(q^{+}\right)+I_{D S}\left(q^{-}\right)\right] / 2$, was calculated from the $I_{D S}\left(q^{+}\right)$and $I_{D S}\left(q^{-}\right)$values measured for the corresponding positions of a sample in the right and left regions near the exact Bragg position. The antisymmetrical part of DS was determined as $I_{A n t}=$ $=\left[I_{D S}\left(q^{+}\right)-I_{D S}\left(q^{-}\right)\right] / 2$. The average radius $r_{0}$ of microdefects was obtained by the known method of determination of this value from the intersection point $q_{0}$ with the abscissa coordinate of the function i $I_{D S}{ }^{s y m}=f(q)$. Besides, these parameters were also determined by the fitting procedure of calculated reflectivities to the CDR as well as from the maps of isodiffuse intensity contours in a reciprocal space. 


\section{L.I. Datsenko et al.: Complex diffractometrical investigation of structural and ...}

The newly developed experimental method for the independent determination of defect structure parameters from the QFRs total integrated reflectivities, $R_{i}(\lambda)$, for the Bragg diffracted X-rays with wavelengths $l$ situated between the absorption K-edges of sublattice atoms was used, too. In this case the SCS was used. So the integrated reflectivities, $R_{i}(\lambda)=R_{i c o h}(\Delta \theta)+R_{i D}(\Delta \theta)$, for every $\lambda$ consisting of the coherent, $R_{i \operatorname{coh}}(\Delta \theta)$, and diffuse, $R_{i D}(\Delta \theta)$, components were calculated according to (3-5) for the (200) QFR afrer integration these components over the interference region $\Delta \theta$. In the case of a real crystal, both components depend on the Debye-Waller static factor, $E=\exp \left(-L_{H}\right)$, on the extinction coefficients $\mu_{d}$ and $\mu_{d}{ }^{*}$ due to scattering of radiation on defects as well as on the nonstoichiometry parameter $\Delta$. The known Honl corrections for atomic formfactors $f_{0}$ due to anomalous dispersion $\Delta f_{r}^{\prime}$ and $\Delta f_{i}^{\prime \prime}$ were made by a fitting procedure of the calculated $R_{i}(\Delta \theta)$ values (3-5) to the measured ones. Finally the looked for data of the $\bar{r}$ and $\bar{n}$ parameters were obtained taking into account their connection with the mentioned diffraction characteristics, $L_{H}, \mu_{d s}$ and $\mu_{d s}{ }^{*},[1,18]$ (Table 1).

Silicon concentration, $n_{S i}$, in the GaAs films was determined by means of the secondary ion mass spectroscopy (SIMS) to be $2.5 \cdot 10^{19}$ and $1.6 \cdot 10^{20} \mathrm{~cm}^{-3}$ for the samples 1 and 2 , respectively. These values exceeded considerably the concentration $p$ of charge carriers (holes) $p=$ $=2 \div 5 \cdot 10^{18} \mathrm{~cm}^{-3}$. The difference between two values testified for large quantity of silicon atoms situated in an electrically passive interstitial positions. Thickness of the films 1 and 2 were rather large, i.e. 13 and $15 \mu \mathrm{m}$, which provided possibility to register only the reflectivities from the epitaxial films, but not taking into account scattering from substrates.

\section{Results and discussion}

Let us consider firstly the experimental results concerning the fundamental reflection (400) (points in the Fig. 1) where the calculated rocking curve (CDR) 1 is shown for the sample 1 . The coherent (2) and diffuse (3) components of the CDR are given, too. Diffuse component of the CDR gives main contribution in scattering both at the left and right parts of the curve. This component of scattering has some minimum at $\Delta \theta=0$ due to dynamical interaction of diffuse scattering with crystalline matrix (extinction phenomenon). Contribution of diffuse scattering here should be taken into account to obtain the correct value of total intensity which agrees satisfactory with experimental points. One can note relatively good agreement between the experimental and calculated results obtained by corresponding formulae of the dynamical theory of scattering by homogeneously distributed Coulomb deformation centres $[14,19]$. One should note here that the formulae of this theory describe properly the DS component behaviour in the diffraction maximum region both for the small $(r<\Lambda)$ as well as large $(r>\Lambda)$ defect cases ( $\Lambda$ is an extinction length distance), too.

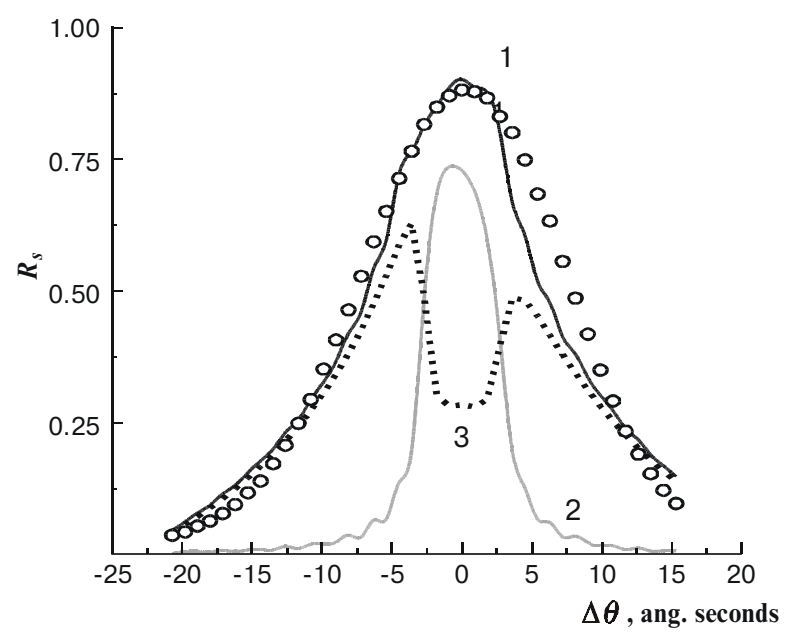

Fig. 1. Experimental (points) and theoretical (solid line) diffraction rocking curves for the sample 1 obtained at $\Delta \theta^{\prime}=0$ position. The coherent and diffuse intensity components are denoted by 2 and 3 , curves respectively. All values are normalized by the intensity corresponding to the exact Bragg position. (400) reflection of $\mathrm{CuKa}_{1}$ radiation.

One can judge about the DS component level in one of the samples from the Fig. 2 where the curve 1 taken for the (400) reflection corresponds to the case of the diffraction peak as registered without a crystal-analyser. The tails of the curve 1 due to DS are rather high as comparing with those in the curve 2 that was registered with analyzer.

The characteristics of microdefects in two GaAs:Si/ GaAs films $\left(r_{01}\right.$ and $\left.n_{01}\right)$ determined by fitting procedure of the theoretical CDR curves to the experimental points are given in Table 1. The average radius of microdefects $r_{01}$ happened to be close to the value $r_{02}=1.5 \mu \mathrm{m}$ calcu-

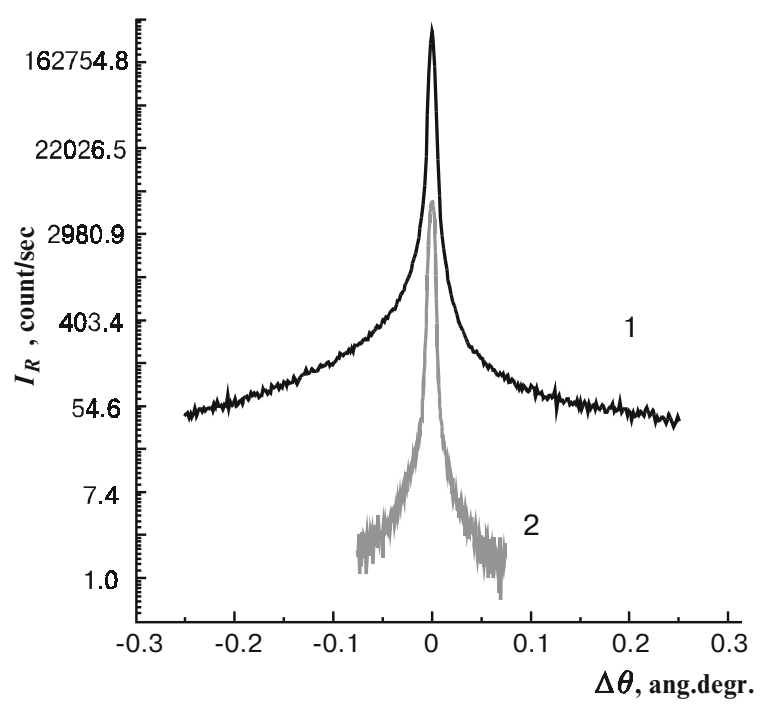

Fig. 2. The level of the (400) rocking curves DS tailes in the sample 1 obtained without crystal-analyser (1) and with it (2). 


\section{L.I. Datsenko et al.: Complex diffractometrical investigation of structural and ...}

Table 1. Structural characteristics $\left(r_{0}, n_{0}\right)$ of precipitates in GaAs films with $\mathrm{Si}$ as determined by the two independent methods as well as the nonstoichiometry parameter $\Delta$.

\begin{tabular}{c|c|c|c|c|c}
\hline \hline $\begin{array}{c}\text { Number } \\
\text { of the sample }\end{array}$ & \multicolumn{2}{|c|}{$\begin{array}{c}\text { Fitting of the theoretical } \\
\text { CDR to experimental ones, } \\
(400) \text { reflection }\end{array}$} & \multicolumn{3}{|c}{$\begin{array}{c}\text { Spectral dependence of reflectivity, } \\
R_{i}(\lambda),(200) \text { reflection [16] }\end{array}$} \\
\hline & $r_{01}, \mu \mathrm{m}$ & $n_{01}, \mathrm{~cm}^{-3}$ & $r_{03}, \mu \mathrm{m}$ & $n_{03}, \mathrm{~cm}^{-3}$ & $\Delta=C_{\mathrm{Ga}}-C_{\mathrm{As}}$ \\
\hline 1 & 2.5 & $5.0 \cdot 10^{6}$ & 1.0 & $2.5 \cdot 10^{6}$ & 0.047 \\
\hline 2 & 2.0 & $1.5 \cdot 10^{8}$ & 1.8 & $2.0 \cdot 10^{8}$ & 0.008 \\
\hline \hline
\end{tabular}

Note: The values of microdefect radii $r_{02}$ determined also from the intersection point $q_{0}$ of the $I_{D S} S^{s y m}=f(q)$ function with abscissa axis [18] are respectively 1.5 and $2.1 \mu \mathrm{m}$ for the samples 1 and 2 .

lated from the of scattering vector value $q_{0}$ as determined from the graph for differential DS reflectivity $R_{D S}=$ $f(\ln \Delta \vartheta)$. At last, these parameters of microdefects, i.e. $r_{03}$ and $n_{03}$, have been independently obtained by the abovementioned method of registration of $R_{i}(\lambda)$ spectral distribution [16] by a SCS (Table 1).

Satisfactory agreement between all three independent experimental values of radius $r_{0}$ and concentration $n_{0}$ of microdefects, obtained with utilization of the new analytical relations between the differential diffuse as well as coherent scattering components of the intensities, both integrated over the Ewald sphere [14,19], and characteristics of microdefect structure testifies that the mentioned theory describes properly interaction of X-rays with the films studied.

The plots of the $\ln R_{D S}=f(\ln \Delta \vartheta)$ function for the differential DS showed the slope of the graph to be close to 2 . It means that experimental results were obtained just in the Huang region of scattering [18]. We have not obtained the slope value close to 5 . It could possibly indicate that our films did not contain linear dislocations [20]. The law of antisymmetrical part of DS variations with $q$ was close to the theoretical regularity (the tangent of slope angle was $\sim 1,3$ ). As far as the antisymmetrical part of the DS had larger level at the positive region of $q$ variation, one can draw the conclusion that the predominate type of microdefects in $\mathrm{GaAs}$ (Si) films had an interstitial character (precipitates).

One of the experimental maps of the isodiffuse countors (two-dimensional distribution of intensity near (400) RELP together with above-mentioned «resolution stars» due to the coherent part of the $\mathrm{CuKa}_{1}$ scattering) is shown for the sample 2 in Fig. 3a. The characteristic minimum of the intensity distribution known for the case of spherical clusters was not possible to be observed because of presence of the mentioned coherent component
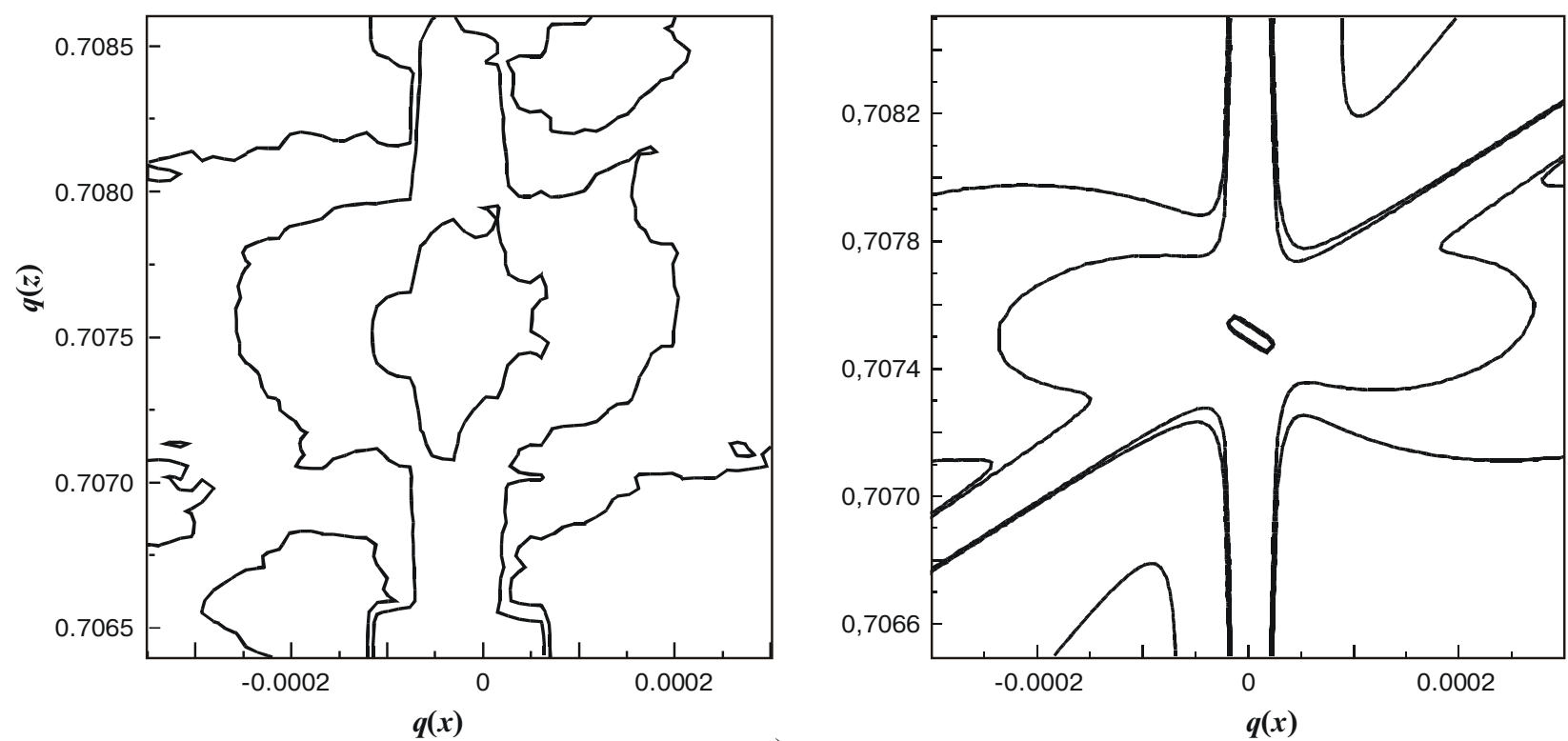

a)

Fig. 3. Experimental (a) and calculated (b) contours of the lines of equal intensity situated closely to the (004) RELP for the sample $\mathrm{N} 2 . \mathrm{CuKa}_{1}$ radiation. The contour levels are 10000, 5000, 100 and 5 counts s $\mathrm{s}^{-1}$. 

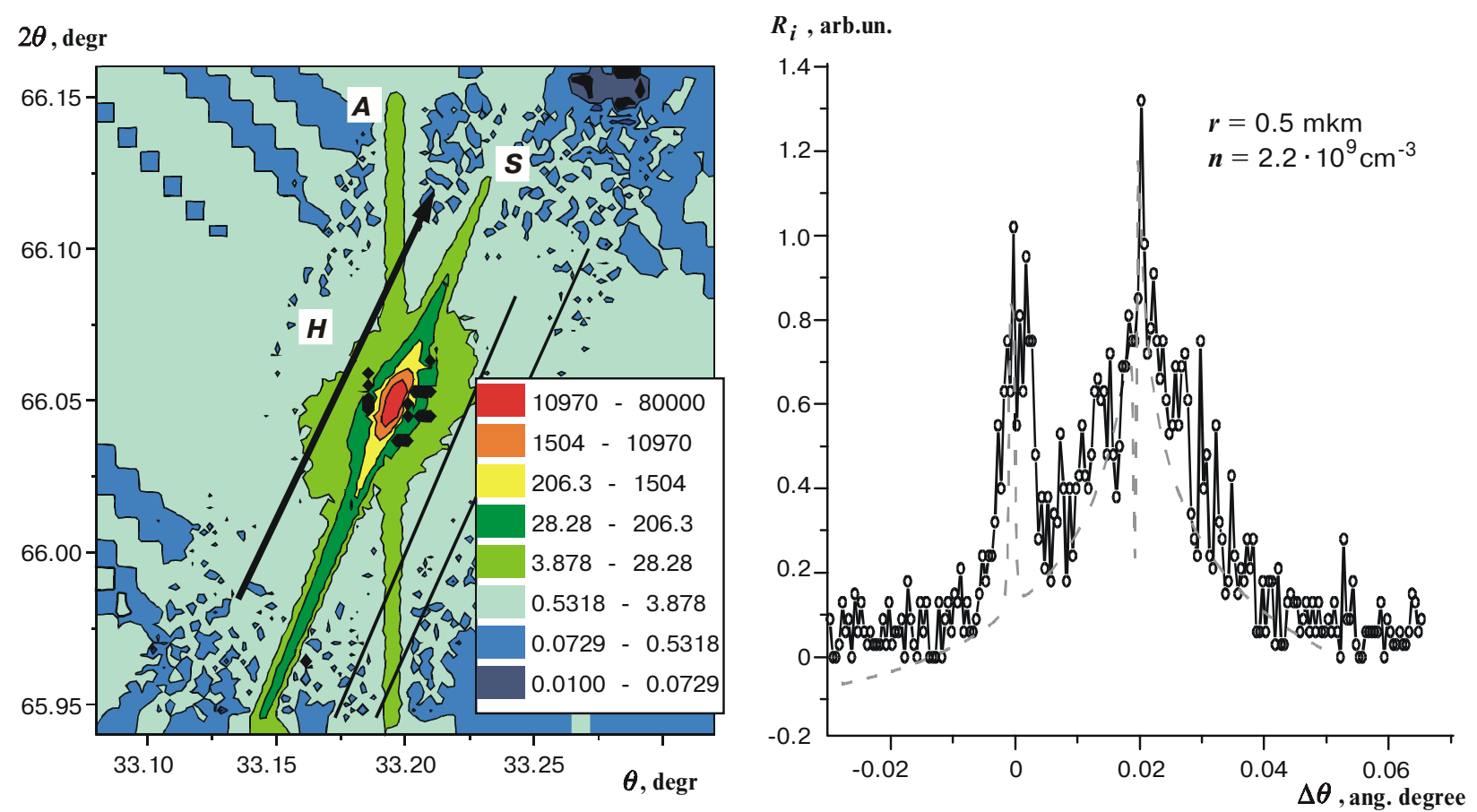

Fig. 4. The experimental map of intensity distribution near the (400) fundamental RELP (a) for the sample 3 and one of the section (triangles) taken at $\left(\Delta \vartheta^{\prime}=0.03^{0}\right)$ (b). The result of calculation for $r=0.38 \mu \mathrm{m}$ and $n=5.5 \cdot 10^{9} \mathrm{~cm}^{-2}$ are depicted by the dotted lines. The levels of intensity contours are shown in the insert in (a).

of scattering. The corresponding theoretical map (Fig. 3b) as calculated using formulae (1-6) in [17] is in qualitative agreement with the experimental ones (Fig. 3a).

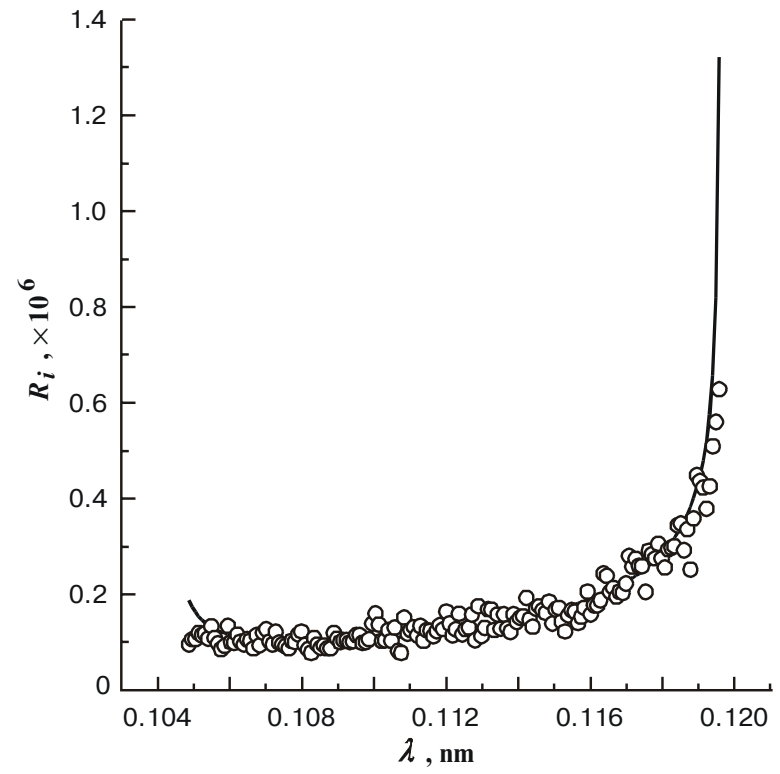

Fig. 5. Experimental (markers) and calculated (solid line) values of the integrated reflectivities $R_{i}$ of the (200) QFR as a function of wavelengths of continuous spectrum situated between $\mathrm{Ga}$ and As absorption $K$-edges of the spectrum as calculated using the Molodkin dynamical theory [14].
However, one can compare quantitatively different sections of a measured map. One of such section position as noted conditionally by thin lines parallel to the diffraction vector $\mathbf{H}$ direction in the experimental map (Fig. 4a) taken from the another sample 3 is shown in Fig. 4b. The average values of the radius $\bar{r}$ and concentrations $\bar{n}$ of precipitates obtained by fitting procedure for the two section positions of this sample are, respectively, $0.83 \mu \mathrm{m}$, $3.2 \cdot 10^{8} \mathrm{~cm}^{-3}$ and $0.38 \mu \mathrm{m}, 5.5 \cdot 10^{9} \mathrm{~cm}^{-3}$. The first group of parameters (larger radius and relatively lower concentration of defects) determines the scattering closer to the (400) RELP. The second group of parameters describes the value of intensity further of it.

Now let us discuss the data obtained from the twodimensional TCS maps and those determined using analysis of reflectivity spectral dependence (Fig. 5) [16] for the sample 3 . The first method gave the following values of $r=3.5 \mu \mathrm{m}$ and $n=4.3 \cdot 10^{6} \mathrm{~cm}^{-3}$ whereas some another parameters $\left(r=4.8 \mu \mathrm{m}\right.$ and $\left.n=9.4 \cdot 10^{6} \mathrm{~cm}^{-3}\right)$ were obtained from fitting of the calculated integral reflectivities to the measured data of $R_{i}(\lambda)$. It seems to us that both independent methods gave close data. The method [16] enabled to determine also the level of nonstoichiometry $\Delta=0.009$ (Ga excess) in this sample.

\section{Conclusion}

Summarizing the main results of this paper one can note that the complex of X-ray diffractometrical methods for analysis of diffuse scattering peculiarities in GaAs films 


\section{L.I. Datsenko et al.: Complex diffractometrical investigation of structural and ...}

heavily doped with silicon enabled to discover the microdefects (Coulomb deformation centers) of different sizes and concentrations. By means of the X-ray high resolution diffractometry (the Philips three-crystal diffractometer) the defect structure parameters have been determined. They happend to be close to these values as determined by independent methods. One of them is the newly developed method for analysis of reflectivities $R_{i}(\lambda)$ for quasi-forbidden Bragg reflections of radiation with wavelengths situated between the absorption K-edges for atoms of sublattices.

The agreement between the experimental and calculated maps of isodiffuse contours of intensities for usual (fundamental) and quasi-forbidden reflections has the qualitative character only maybe due to complex interstitial nature of defects. Quantitave agreement between the measured and calculated data was established for the angular intensity distribution sections, i.e. for rocking curves, as well as for the sections of the experimental maps. The quantity of silicon atoms in GaAs (Si) films evaluated from the values of average radius and concentration of microdefects is close in order of magnitude to concentration of this impurity as determined by the method of secondary ions mass spectroscopy.

\section{References}

1. M. A. Kryvoglaz, Diffraction of X-ray and thermal neutrons in imperfect crystals, Springer, Berlin; Heidelberg, p.473 (1992).

2. B. C. Larson // J.Appl.Cryst. 8, p.150 (1975).

3. W. Mayer, D. Grasse and J.Peisl // Phys. Stat Sol.(a)., 87, p.583 (1985)

4. P. Ehrhard and W. Schilling // Phys.Rev. (B). 8, p.2604 (1973).
5. H. Trinkaus // Phys.Stat.Sol.(b). 51, p.307 (1972).

6. H. Trinkaus // Phys.Stat.Sol.(b). 54, p.209 (1972).

7. H. Peisl, Structural information and defect energies studies by X-ray methods. In «Defects and their structure in nonmetallic solids». Edited by B.Henderson and A. E. Hughes // Plenum Publishing Corporation, New-York p.381 (1976).

8. R. N. Kutt // Soviet solid state physics, (in Russian) 31, p.270 (1989).

9. R. N. Kutt // Soviet solid state physics, (in Russian) 39, p.1188 (1997).

10. U. Bonse, E. Kappler and A. Schill, Z. Phys. 178, p.221 (1964).

11. B. C. Larson and W. Schmatz // Phys.Rev.(B). 10, p.2307 (1974).

12. P. Eisenberger, N. G. Alexandropoulos and P. M. Platzmann // Phys.Rev.Lett. 28, p.519 (1972).

13. V.V. Ratnikov, L.M. Sorokin and G. N. Mosina, Soviet solid state physics, (in Russian) 23, p.58 (1981).

14. V.B. Molodkin, V.V. Nemoshkalenko, S.I. Olikhovskii, E.N. Kislovskii, O.V. Reshetnyk, T.A. Vladimirova, V.P. Krivitsky, V.F. Machulin, I.V. Prokopenko G.E. Ice and B.C. Larson // Metal Physics and Advanced Technologies, 18, p.1239 (1998).

15. M.G. Milvidski and V.B. Osvenskii, Structurnye defecty v monocrystallakh poluprovodnikov // Metallurgiya publishing company, Moscow, p. 256 (1984).

16. L.I. Datsenko, V.P. Klad'ko, V.F. Machulin, S. Manninen, I.V. Prokopenko, Dynamical scattering of X-rays by real crystals and problem of point defects. In «Theory and computation for synchrotron radiation spectroscopy», Frascati, Italy 1999, Ed. by M. Benfatto, C. R. Natoli, E. Pace // AIP Conference Proceedings, p.514.

17. V.P. Klad'ko, L.I. Datsenko, J. Bak-Misiuk, S.I. Olikhovskii, V.F. Machulin, I.V. Prokopenko, V.B. Molodkin, Z.V. Maksimenko. J.Phys.D: Appl.Phys., 34, p.A87. (2001).

18. P. H. Dederichs // Phys.Rev.(B)., 4, p.1041 (1971).

19. B. Molodkin S. I. Olikhovskii, E. N. Kislovskii, V. P. Krivitskii., A. V. Los', E. V. Pervak, G. E. Ice and B. C. Larson // Metal Physics and Advanced Technologies, 17, N2, p.1383 (1997)

20. V.T. Bublik and K.D. Shcherbachov, (in Russian) //Kristallografiya. 39, p.1105 (1994). 